This is the post peer-review accepted manuscript of:

F. Guidi, A. Guerra, D. Dardari, A. Clemente and R. D'Errico, "Environment Mapping with Millimeter-Wave Massive Arrays: System Design and Performance," 2016 IEEE Globecom Workshops (GC Wkshps), Washington, DC, 2016, pp. 1-6.

https://doi.org/10.1109/GLOCOMW.2016.7848895

The published version is available online at:

http://ieeexplore.ieee.org/stamp/stamp.jsp?tp=\&arnumber=7848895\&isnumber=7848793

(C) 2016 IEEE. Personal use of this material is permitted. Permission from IEEE must be obtained for all other uses, in any current or future media, including reprinting/republishing this material for advertising or promotional purposes, creating new collective works, for resale or redistribution to servers or lists, or reuse of any copyrighted component of this work in other works 


\title{
Environment Mapping with Millimeter-wave Massive Arrays: System Design and Performance
}

\author{
Francesco Guidi ${ }^{\dagger \ddagger}$, Anna Guerra*, Davide Dardari*, Antonio Clemente ${ }^{\dagger \ddagger}$ and Raffaele D'Errico ${ }^{\dagger \ddagger}$ \\ ${ }^{\dagger}$ CEA, LETI, MINATEC Campus, 38054 Grenoble, France \\ $\ddagger$ Univ. Grenoble-Alpes, 38000 Grenoble, France \\ \{francesco.guidi, antonio.clemente, raffaele.derrico\}@cea.fr \\ *DEI, University of Bologna, via Venezia 52, I-47521 Cesena (FC), Italy \\ \{anna.guerra3, davide.dardari\}@unibo.it
}

\begin{abstract}
Recently, several studies have been moving towards the idea to adopt massive arrays for localization and mapping applications thanks to the high scanning resolution achievable. In this framework, this paper investigates the impact of different design parameters in order to maximize the mapping performance while keeping the receiver architecture as simple as possible when a Bayesian approach is used. To this end, thresholding strategies to filter out unwanted received signal components (cleaning operation before soft mapping), e.g. thermal noise, are analyzed together with other design parameters that can operate directly on raw data. Considerations about possible system design configurations are then drawn inspired by results obtained using measured data, which showed a more pronounced impact of the cleaning approach for $1 \mathrm{GHz}$ bandwidth with respect to $3 \mathrm{GHz}$.

Index Terms-Millimeter-waves, massive antenna arrays, indoor mapping, personal radar.
\end{abstract}

\section{INTRODUCTION}

Nowadays, several studies are focusing towards the miniaturization and the adoption of large scale arrays [1] for several ranging and localization applications, such as mobile communication [2], simultaneous localization and mapping (SLAM) [3], [4] or personal radars [5], thanks to the possibility to achieve a precise and high-scanning resolution given by the large number of adopted antennas [6]-[9].

In traditional SLAM, a robot equipped with a laser-based radar or a camera (visual SLAM) moves in an unknown indoor environment and is able to reconstruct a map of it while inferring its own position and orientation inside the generated map [3]. The main shortcomings of traditional approaches are mainly related to technological aspects. Indeed, laser-based radar as well as camera-based systems work well only in perfect visibility conditions and they require a mechanical steering in order to perform a scanning operation [10]. Instead, when moving towards human-centric systems, as for example personal radars [5], electronic steering capabilities are highly appreciated to reduce the user active participation to the mapping process and to overcome the shortcomings of laser.

When considering millimeter-wave $(\mathrm{mmW})$ massive arrays, different technological issues could arise. In fact, a tradeoff has to be met to assure electronic steering capabilities, high directivity, low side-lobes as well as to maintain the array complexity and cost low. In [5], [11], [12], mapping

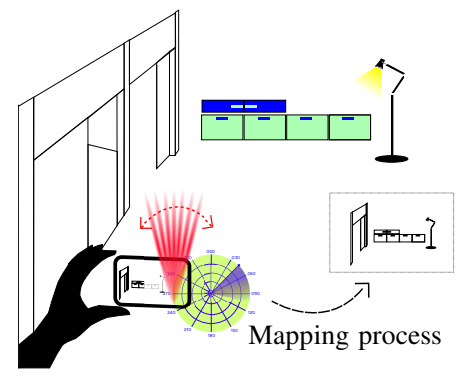

Fig. 1. Personal radar scenario.

performance with massive arrays have been investigated as a function of the array characteristics and errors.

Motivated by this background, in this paper we exploit real measured data to investigate and propose different techniques to ameliorate the mapping capabilities of a low complexity and cost non-coherent receiver, based on $\mathrm{mmW}$ massive arrays, for personal radars applications. In our specific scenario, a unique receiver section is considered where the signals received at each antenna branch are properly combined by phase shifters blocks. We propose also a cleaning operation in which the threshold strategy is intended to improve the mapping performance. By investigating the impact of different design parameters, we show that environment reconstruction can become more reliable.

The rest of the paper is organized as follows. Sec. II describes the system model whereas Sec. III reports some details related to the mapping algorithms considered, and some of the key parameters involved for the design of the algorithm. Finally, in Sec. IV mapping results are reported and commented. Sec. V concludes the work.

\section{SYSTEM MODEL}

We consider a personal radar in monostatic configuration, i.e. with TX and RX sections co-located, which moves in the surrounding space and, for each position, it performs a scanning operation by steering its beam in different $N_{\text {steer }}$ directions. For each steering direction $\theta_{b}$, a train of $N_{\mathrm{p}}$ pulses with bandwidth $W$ is transmitted. 


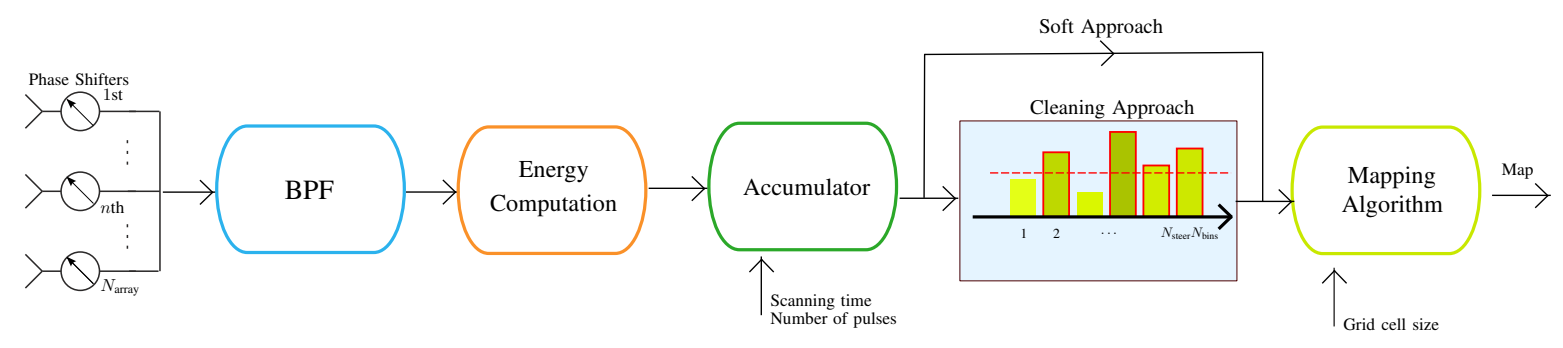

Fig. 2. Considered receiver scheme.

To perform radar-like operations, we consider a noncoherent approach based on energy measurements, according to the receiver architecture reported in Fig. 2. As it can be noticed, the received signals at each antenna branch are properly combined by phase shifters blocks to maximize the array gain in a particular direction in space. Then the collected responses are filtered to eliminate the out-of-band noise. From the filtered received signals, energy bins are evaluated during a time frame $T_{\mathrm{f}}$ after the transmission of each pulse by considering $N_{\text {bins }}=\left\lfloor T_{\mathrm{f}} / T_{\mathrm{ED}}\right\rfloor$ time slots (bins) of duration $T_{\mathrm{ED}} \approx 1 / W$. Energy measurements are accumulated for each time bin over the $N_{\mathrm{p}}$ frames of the interrogation signal. The accumulated measured energy vector, containing the values for all the steering directions at the output of the receiver, is defined as

$\mathbf{e}=\left[e_{11}, \ldots, e_{1 N_{\text {bin }}}, \ldots, e_{b s}, \ldots, e_{N_{\text {steer }} 1}, \ldots e_{N_{\text {steer }} N_{\text {bin }}}\right]^{\mathrm{T}}$

where $[\cdot]^{\mathrm{T}}$ indicates the transpose operation and $e_{b s}$ is given by

$$
e_{b s}=\sum_{l=0}^{N_{\mathrm{p}}-1} \int_{(s-1) T_{\mathrm{ED}}}^{s T_{\mathrm{ED}}} y^{2}\left(t+l T_{\mathrm{f}}, \theta_{b}\right) d t
$$

with $s=1,2, \ldots, N_{\text {bin }}, b=1,2, \ldots N_{\text {steer }}$ and where we have indicated with $y(\cdot)$ the received filtered signal.

Then, two different steps can be followed: (i) a soft decision mapping (Fig. 2-top), where data are directly included in the mapping algorithm; (ii) a cleaning procedure on the measured energy vector in order to limit the impact of unwanted components in the mapping approach. Thus, based on these two different approaches, it is possible to infer the map of the explored environment by exploiting the algorithm defined in Sec. III.

\section{MAPPING ALGORITHM}

In the considered approach, measured energy bins are included in the mapping algorithm. In particular, we consider the grid-based state-space model for probabilistic mapping in [11], where performance is investigated by means of real measured data. To this purpose, we assume that the radar orientation and trajectory are known.

We represent the environment with a grid of $N_{\mathrm{L}}=X_{\text {grid }} Y_{\text {grid }}$ cells, where each cell is associated with a root radar cross section (RRCS) value to be inferred by the estimation process. ${ }^{1}$

\footnotetext{
${ }^{1}$ We choose to estimate the RRCS (with sign) instead of the radar cross section (RCS) because we model it as a Gaussian random variable.
}

As it will be described later, the choice of cell size impact both the mapping accuracy and the computational complexity.

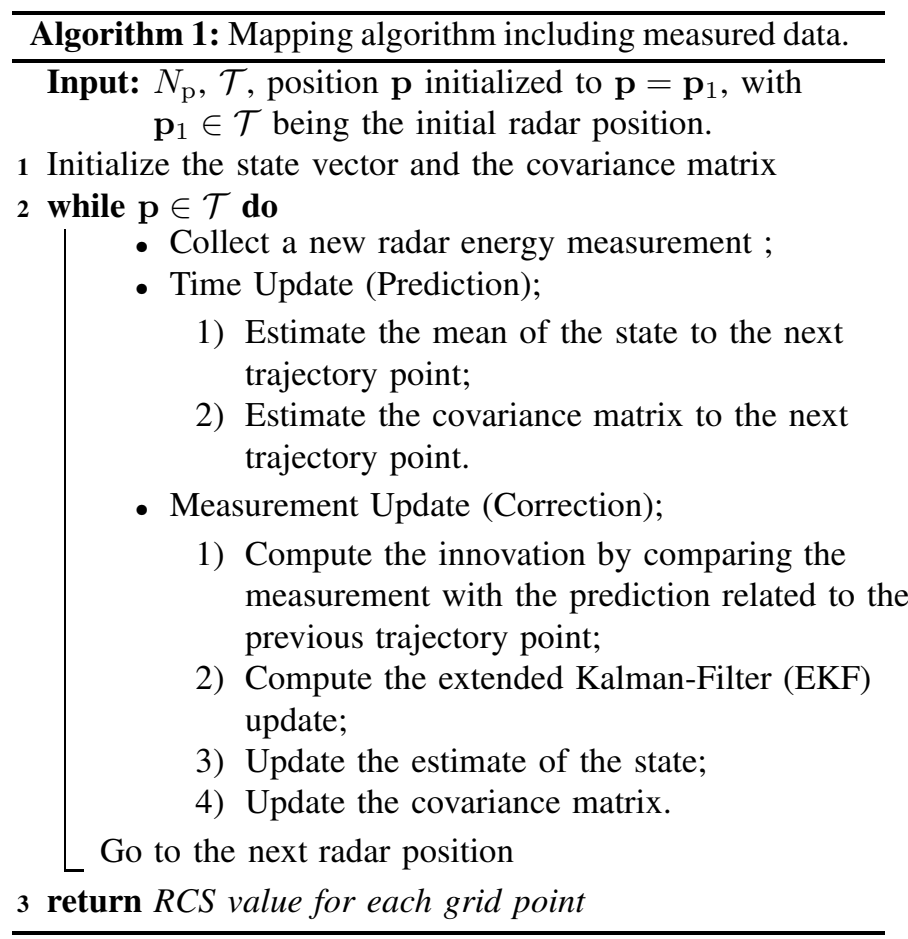

The mapping algorithm is reported in Algorithm 1, where we considered $\mathcal{T}=\left[\mathbf{p}_{1}, \ldots, \mathbf{p}_{N_{\mathrm{POS}}}\right]$ the vector containing the $N_{\text {POS }}$ radar positions (i.e. the spatial coordinates) inside the room. Moreover, since we accounted for a stationary environment, the transition model is neglected. In particular, we assume the following state vector of the system

$$
\mathbf{x}(k)=\mathbf{m}(k)=\left[m_{1}(k), \ldots, m_{i}(k), \ldots, m_{N_{\mathrm{L}}}(k)\right]^{\mathrm{T}}
$$

where $k$ is the discrete time instant and $m_{i}(k)$ indicates the RRCS of the $i$ th cell of the grid, where the frequency dependency has been neglected. In case a soft mapping approach is considered, the mapping process is not preceded by a detection phase related to the vector $\mathbf{e}(k)$ containing the accumulated measured energy at the output of the receiver at time $k$. The corresponding Gaussian observation model $\mathbf{z}(k)$ is fully described in [5].

According to [5], [13], the map estimation process is performed by adopting the EKF method to efficiently evaluate 
the posterior distribution $p(\mathbf{x}(k) \mid \mathbf{e}(1: k))$ of $\mathbf{x}(k)$ given the set of measurements $\mathbf{e}(1: k)=\{\mathbf{e}(1), \mathbf{e}(2), \ldots, \mathbf{e}(k)\}$, from which a maximum a posteriori estimate of the state $\mathbf{x}(k)$ is derived.

Differently from the literature, here a detection phase is not accounted for in the mapping process. In the following, we briefly describe a simple approach to reduce the impact of noisy bins into the algorithm herein reported.

\section{A. Cleaning of Measured Data}

Define now, for each energy bin, the energy detector test

$$
\Lambda_{b s}=e_{b s} \underset{\mathcal{D}_{0}}{\stackrel{\mathcal{D}_{1}}{\gtrless}} \xi_{b s}
$$

where $\xi_{b s}$ being the threshold for the $b$ th steering direction and $s$ th bin, and $\mathcal{D}_{1}$ and $\mathcal{D}_{0}$ represent the two states in which the target in the $\theta_{b}$ steering direction and for the $s$ th time bin overcomes or not the threshold, respectively. The presented decision rule consists in

$$
\text { Assign: }\left\{\begin{array}{lll}
P_{w} T_{\mathrm{f}}, & \text { if } & \mathcal{D}_{0} \\
e_{b s}, & \text { if } & \mathcal{D}_{1}
\end{array}\right.
$$

where $P_{w}=\frac{\sigma^{2} T_{\mathrm{ED}} N_{\mathrm{p}}}{T_{\mathrm{f}}}$ is the power noise. Contrarily to classical detection schemes, where the bins below the threshold are discarded, here they are assigned to a fix value to let the mapping algorithm to converge quickly, as $P_{w} T_{\mathrm{f}}$ represents the initialization value of the cells. To mask noisy bins, the threshold can be designed accounting for a global requirement on the probability of false alarm (PFA) $P_{\text {FA }}^{\star}$ for the $b$ th steering direction, which can be written as

$$
P_{\mathrm{FA}}^{\star}=1-\prod_{s=1}^{N_{\text {bins }}}\left(1-p_{b s}^{(\mathrm{FA})}\right) \approx N_{\text {bins }} \cdot p_{\mathrm{FA}}
$$

where it is assumed that all bins $N_{\text {bins }}$ are statistically independent for a steering direction, and $p_{b s}^{(\mathrm{FA})} \ll 1$. Consequently, the required $p_{\mathrm{FA}}^{\star}$ per bin can be expressed as

$$
p_{\mathrm{FA}}^{\star} \approx \frac{P_{\mathrm{FA}}^{\star}}{N_{\text {bins }}}
$$

which gives a constant threshold per bin given by

$$
\xi_{b s}=\xi=2\left[\operatorname{Inv} \tilde{\Gamma}\left(\frac{N}{2}, \frac{P_{\mathrm{FA}}^{\star}}{N_{\text {bins }}}\right)\right]
$$

where $\operatorname{Inv} \tilde{\Gamma}(\cdot, \cdot)$ is the inverse gamma regularized function, and $N=N_{\mathrm{p}} 2 W T_{\mathrm{ED}}$ is the number of degrees of freedom. Note that with such approach, the threshold does not depend on the bin and steering index, i.e. $\xi_{b s}=\xi$, and it is set to keep the PFA due to the receiver noise to a desired value $P_{\text {FA }}^{\star}$.

\section{B. Design Parameters Impact}

According to the previously defined mapping algorithm, we have different competing effects entering the mapping algorithm.

A key design parameter is represented by the transmitted number of pulses $N_{\mathrm{p}}$. In fact, the higher it is, the higher will
TABLE I

SUMMARY OF THE TRADE-OFFS CONSIDERED FOR THE MAPPING ALGORITHM.

\begin{tabular}{|c|c|}
\hline$N_{\mathrm{p}}$ & Scanning time vs SNR \\
Cell size & Mapping accuracy vs level of complexity \\
Max Range & Noisy bins vs useful bins discarded \\
$W$ & Temporal resolution vs level of complexity \\
Cleaning Approach & Reduced noisy bins vs level of complexity \\
\hline
\end{tabular}

be the signal-to-noise ratio (SNR) of the accumulated received waveforms. On the other side, increasing too much $N_{\mathrm{p}}$ implies a higher transmitted power. Last but not least, consider the scanning time per position defined as

$$
T_{\text {scan }}=N_{\text {steer }} \cdot T_{\text {ob }}=N_{\text {steer }} \cdot N_{\mathrm{p}} \cdot T_{\mathrm{f}} .
$$

where $T_{\mathrm{ob}}$ is the observation time. If the system is constrained by a certain scanning time, $N_{\mathrm{p}}$ should be preserved as low as possible.

Another possibility is to reduce the maximum range, i.e. the number of bins, with the purpose to avoid the inclusion of noisy bins into the mapping process. Such procedure has to account for the environment characteristics (which are not a-priori known), and thus the parameters should be cautiously changed. In addition, if too many bins are discarded, there is the risk to waste useful source of information. The alternative is to reduce $T_{\mathrm{f}}$ with the problem to have a system affected by inter-frame interference.

As also shown in [5], the bandwidth $W$ impact the mapping resolution, thus if possible it has to be increased with an augmented impact of array non-idealities into the performance.

Another important parameter is the grid size: the lower it is, the more accurate is the environment representation. On the other side, the computational complexity is increased as a more complex state vector has to be updated.

In the numerical results, we briefly investigate how the parameters and techniques impact the performance.

\section{RESUlts}

\section{A. Measurement Campaign}

In order to validate the personal radar concept, a measurements campaign has been conducted at CEA-Grenoble in a corridor, where we adopted transmitarrays (TAs) which are a possible candidate for massive arrays applications [12].

We connected a 4-ports Vector Network Analyzer (VNA) operating in the frequency range $10 \mathrm{MHz}-24 \mathrm{GHz}$ with 2 $\mathrm{mmW}$ converters (frequency range $50 \mathrm{GHz}-75 \mathrm{GHz}$ ) and to 2 linearly polarized TAs (size $20 \times 20,1$ bit, $F / D=0.5$ ) with gain $23.1 \mathrm{dBi}$ [14]. The two TAs were placed in a bistatic configuration on a $X$-Y-Azimuth positioner, spaced apart of $0.16 \mathrm{~m}$ to reduce the antenna coupling and to separate the transmitting and receiving channels. Measurements have been collected in 12 different positions spaced of $0.405 \mathrm{~m}$ in the frequency range between $55-70 \mathrm{GHz}$ with a step of $5 \mathrm{MHz}$. Thanks to mechanical rotations (the TAs exploited for measurements were not electronically reconfigurable), the $X$ $Y$-Azimuth positioner rotates the radar in the semi-plane from 

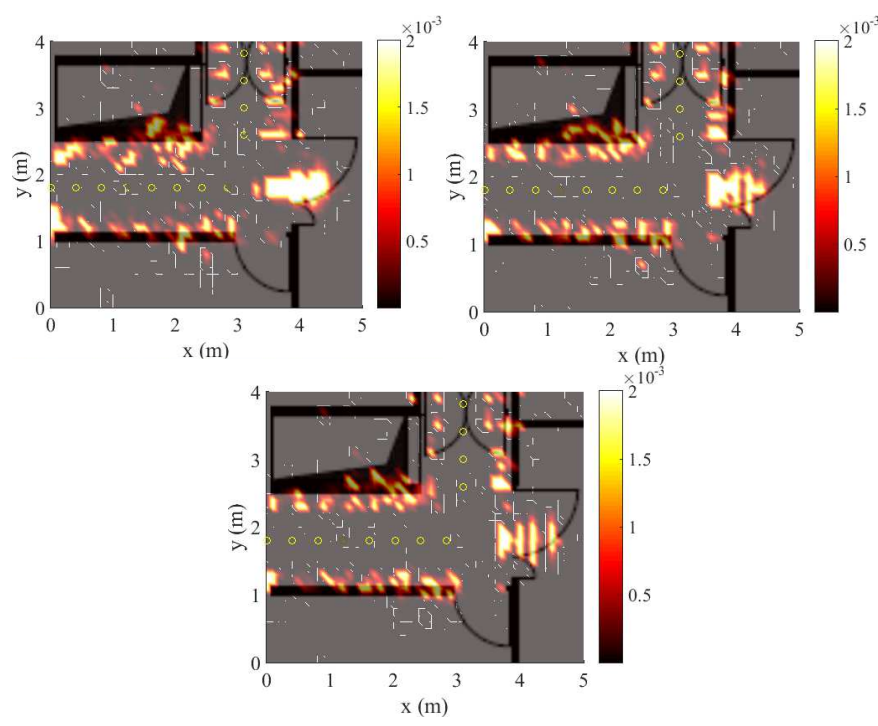

Fig. 3. $W$ impact. RCS results for $N_{\mathrm{p}}=100$, cell size $0.1 \times 0.1 \mathrm{~m}^{2}$ $\mathrm{MR}=15 \mathrm{~m}$ and no cleaning. Top-left: $W=1 \mathrm{GHz}$. Top-right: $W=2 \mathrm{GHz}$. Bottom: $W=3 \mathrm{GHz}$.

$-90^{\circ}$ to $90^{\circ}$ with a step of $5^{\circ}$ according to the TA half power beamwidth (HPBW).

\section{B. Mapping Results}

We now report mapping results using the previously described algorithm, and by varying the parameters of interest in order to investigate the achievable performance. If otherwise indicated, we consider a noise figure of $N_{\mathrm{F}}=4 \mathrm{~dB}, T_{0}=$ $290 \mathrm{~K}$ and a signal bandwidth of $W=1-2-3 \mathrm{GHz}$ (with $T_{\mathrm{ED}}=1 / W$ ). We account for root raised cosine (RRC) pulses centered at frequency $f_{\mathrm{c}}=60 \mathrm{GHz}$ and roll-off factor $\alpha=0.6$, compliant with Federal Communications Commission (FCC) mask at $60 \mathrm{GHz}$ [15] by considering an effective radiated isotropic power (EIRP) of $30 \mathrm{dBm}$. The time frame has been set to $T_{\mathrm{f}}=100 \mathrm{~ns}$, and thus a maximum range $\mathrm{MR}=15 \mathrm{~m}$ is considered.

a) Impact of System Design Parameters: We first report the impact of the bandwidth in the mapping performance, as it was also deeply analyzed in [12]. In fact, Fig.3 shows that by increasing the bandwidth it is possible to improve the mapping resolution and, for example, the contour of walls in the environment.

Then, Fig. 4 reports results for a grid cell size of $0.2 \times$ $0.2 \mathrm{~m}^{2}$. The prize of a reduced complexity is paid in terms of highly degraded map resolution. Consequently, during the design of the system, it is preferable to reduce as much as possible the size of the grid cells. In our specific case, $0.1 \times$ $0.1 \mathrm{~m}^{2}$ has been found as a good compromise.

Successively, we investigate the impact of reducing $N_{\mathrm{p}}$ for different bandwidths. As shown in Fig. 5, the measured SNR could be dramatically affected in certain areas of the perimeter. Consequently, $N_{\mathrm{p}}=100$ represents a good choice to achieve reliable SNR for the considered environment, at the prize of around 3.3 times the scanning time needed for $N_{\mathrm{p}}=30$.
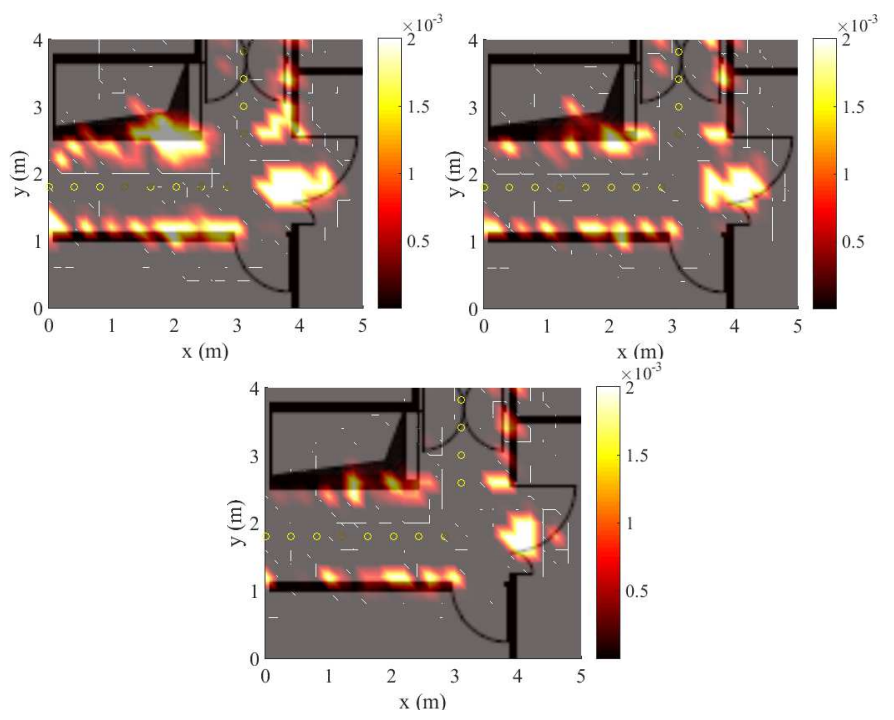

Fig. 4. Cell size impact. RCS results for $N_{\mathrm{p}}=100$, cell size $0.2 \times 0.2 \mathrm{~m}^{2}$, $\mathrm{MR}=15 \mathrm{~m}$ and no cleaning. Top-left: $W=1 \mathrm{GHz}$. Top-right: $W=2 \mathrm{GHz}$. Bottom: $W=3 \mathrm{GHz}$.

Fig. 6 reports instead the impact of the maximum range by discarding the bins after $M R=10 \mathrm{~m}, M R=5 \mathrm{~m}$ or $M R=2 \mathrm{~m}$. Due to the environment chosen for measurements, we experienced a drastic performance improvement only for $M R=2 \mathrm{~m}$. On the other side, it is expected that this parameter could limit the performance in other environments, so its variations should be carefully considered. If from one side part of measured data is wasted by reducing the maximum range, it is not possible to reduce $T_{\mathrm{f}}$ to avoid inter-frame interference, as $T_{\mathrm{f}}$ should be chosen to accomodate the entire backscattered response.

b) Approach with Filtered Energy Values: We successively apply the threshold to measured data, in order to avoid the contribution of undesired components into the mapping algorithm. To this purpose we adopted the following three approaches: (approach 1) exploitation of the threshold $\xi$ derived in (8); (approach 2) the threshold is set by lowering the maximum measured power in $\mathbf{p}_{1}$ of $24 \mathrm{dBm}$; (approach 3 ) the maximum measured power in $\mathbf{p}_{1}$ is lowered of $18 \mathrm{dBm}$. Note that the second and third approaches were conceived in order to mask higher non-ideal radiation pattern effect (i.e. those coming from side-lobes).

Results are reported in Fig. 7 and 8 for the three approaches and different $W$. As it is possible to observe, when the threshold is set to mask the noise only, i.e. with approach 1 , the performance variations with respect to Fig. 3 are not evident. On the other side, by increasing the threshold, i.e. by using approaches (2) and (3), the artifacts are reduced with a rough degradation of the contour of the corridor, especially for $W=3 \mathrm{GHz}$ and approach 3. Consequently, we remark that the cleaning technique is more useful when $W=1 \mathrm{GHz}$, and thus it could be a good compromise for real applications where the bandwidth is limited by the available technology.

In summary, the best option seems to be the exploitation 

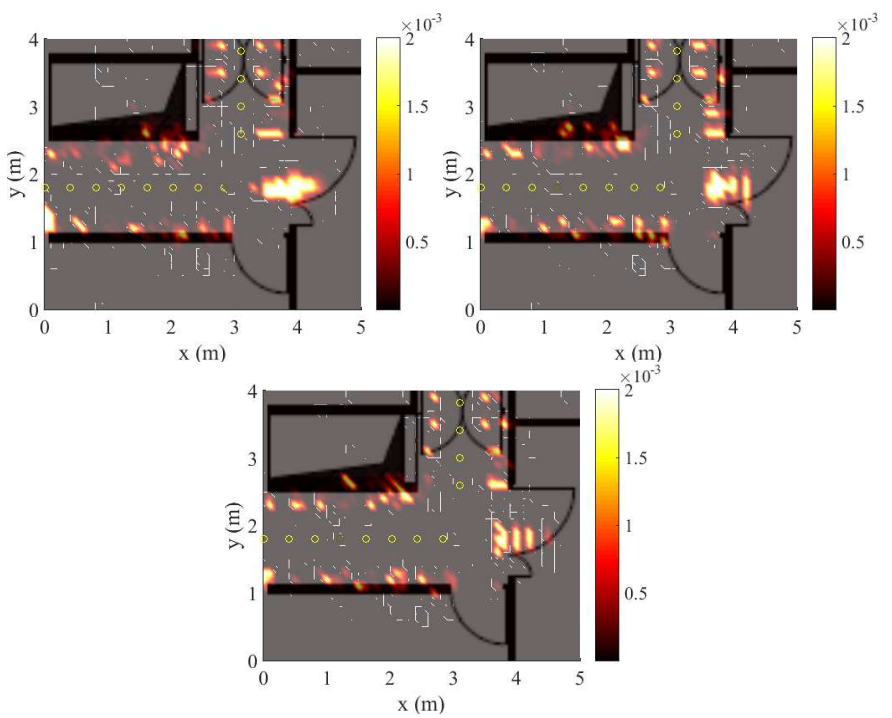

Fig. 5. $\quad N_{\mathrm{p}}$ impact. RCS results for $N_{\mathrm{p}}=30$, cell size $0.1 \times 0.1 \mathrm{~m}^{2}$, $\mathrm{MR}=15 \mathrm{~m}$ and no cleaning. Top-left: $W=1 \mathrm{GHz}$. Top-right: $W=2 \mathrm{GHz}$. Bottom: $W=3 \mathrm{GHz}$.
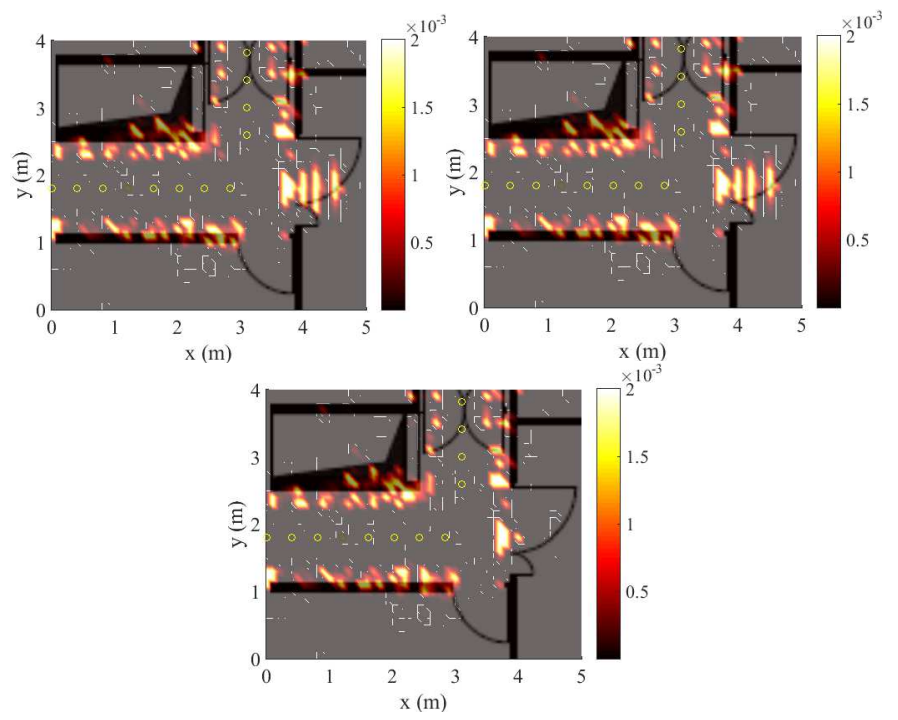

Fig. 6. Maximum range impact for $W=3 \mathrm{GHz}$. RCS results for grid cell size $0.1 \times 0.1 \mathrm{~m}^{2}$ and $N_{\mathrm{p}}=100$. Top-left: $\mathrm{MR}=10 \mathrm{~m}$. Top-right: $\mathrm{MR}=5 \mathrm{~m}$. Bottom: MR $=2 \mathrm{~m}$.

of a $3 \mathrm{GHz}$ bandwidth by limiting the maximum range. On the other side, this solution can work well in the considered scenario, but it could dramatically reduce the map reconstruction in other larger environments. The joint choice of $N_{\mathrm{p}}=100$ with a smaller grid size lets to achieve good mapping performance. The adoption of a threshold seems more indicated whenever a system with $1 \mathrm{GHz}$ is considered. Furthermore, it could be interesting to relate the approaches 2 and 3 herein considered to the massive arrays characteristics, as in part proposed in [16].
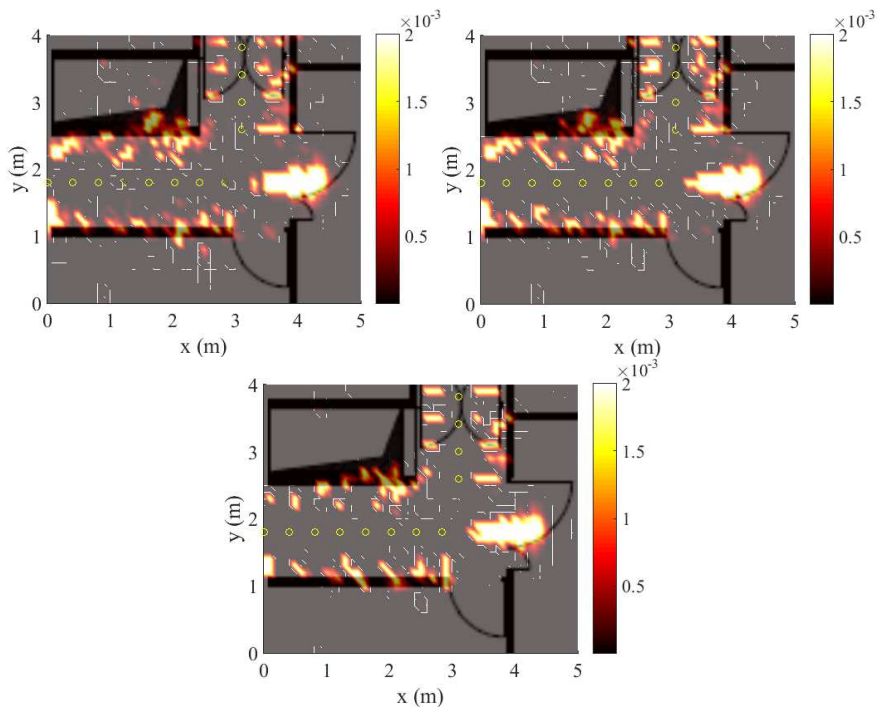

Fig. 7. Cleaning approach for $W=1 \mathrm{GHz}$. RCS results for $N_{\mathrm{p}}=100$, cell size $0.1 \times 0.1 \mathrm{~m}^{2}, \mathrm{MR}=15 \mathrm{~m}$. Top-left: approach 1 . Top-right: approach 2 . Bottom: approach 3 .

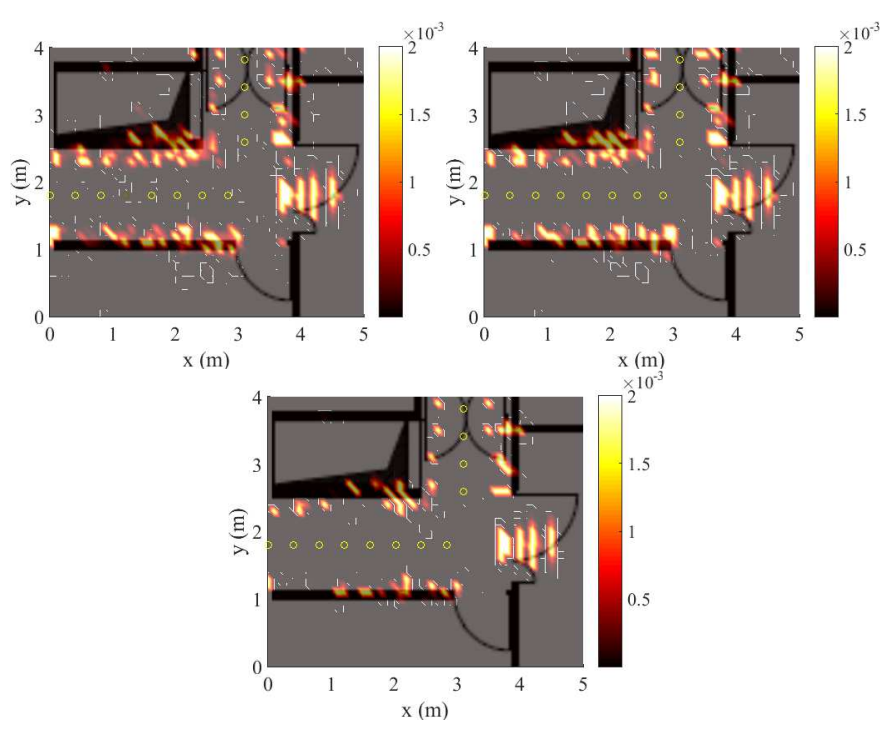

Fig. 8. Cleaning approach for $W=3 \mathrm{GHz}$. RCS results for $N_{\mathrm{p}}=100$, cell size $0.1 \times 0.1 \mathrm{~m}^{2}, M R=15 \mathrm{~m}$. Top-left: Approach 1 . Top-right: approach 2. Bottom: approach 3 .

\section{Conclusions}

In this paper we investigated the impact of different design parameters into the mapping performance when $\mathrm{mmW}$ massive arrays are used for personal radars applications. In particular, by means of measured data and of a grid-based Bayesian statespace approach for map reconstruction, we showed the impact of different design parameters as well as of cleaning approach for measured data. In particular, from the obtained results we experienced that the joint adoption of $W=3 \mathrm{GHz}, N_{\mathrm{p}}=100$ and $0.1 \times 0.1 \mathrm{~m}^{2}$ grid size cells let to achieve good mapping performance, which can be further improved with a reduction of the maximum range MR. On the other side, MR has to be cautiously diminished not to lose useful information from the 
measured energy vectors.

From the obtained results it has also been evidenced that if the bandwidth is limited to $1 \mathrm{GHz}$, a cleaning approach is helpful to improve the performance. To this purpose, enhanced algorithms could be conceived in order to account for the adopted massive arrays characteristics into the algorithm design (i.e. side-lobes).

\section{ACKNOWLEDGMENT}

This work has been supported in part by the H2020EU.1.3.2 IF-EF Marie-Curie project MAPS (Grant 659067) and by the European H2020 project XCycle (Grant 635975).

\section{REFERENCES}

[1] W. Hong et al., "Study and prototyping of practically large-scale mmwave antenna systems for 5G cellular devices," IEEE Commun. Mag., vol. 52, no. 9, pp. 63-69, 2014.

[2] W. Roh et al., "Millimeter-wave beamforming as an enabling technology for 5G cellular communications: theoretical feasibility and prototype results," IEEE Commun. Mag., vol. 52, no. 2, pp. 106-113, 2014.

[3] M. W. M. G. Dissanayake et al., "A solution to the simultaneous localization and map building (SLAM) problem," IEEE Trans. Robot. Autom., vol. 17, no. 3, pp. 229-241, Jun. 2001.

[4] E. Jose et al., "Predicting Millimeter Wave Radar Spectra for Autonomous Navigation,” IEEE Sensors J., vol. 10, no. 5, pp. 960-971, May 2010.

[5] F. Guidi, A. Guerra, and D. Dardari, "Personal mobile radars with millimeter-wave massive arrays for indoor mapping," IEEE Trans. Mobile Comp., vol. 14, no. 99, 2015.

[6] K. Witrisal et al., "High-accuracy localization for assisted living," IEEE Signal Processing Mag., 2016.

[7] X. Huang and Y. Guo, "Frequency-domain AoA estimation and beamforming with wideband hybrid arrays," IEEE Trans. Wireless Commun., vol. 10 , no. 8, pp. 2543-2553, Aug. 2011.

[8] J. Zhang et al., "Massive hybrid antenna array for millimeter-wave cellular communications," IEEE Wireless Commun., vol. 22, no. 1, pp. 79-87, Feb. 2015.

[9] A. Clemente et al., "Wideband 400-element electronically reconfigurable transmitarray in X band," IEEE Trans. Antennas Propag., vol. 61, no. 10, pp. 5017-5027, Oct 2013.

[10] E. Jose and M. Adams, "An augmented state SLAM formulation for multiple line-of-sight features with millimetre wave radar," in Proc. IEEE/RSJ Int. Conf. Intelligent Robots and Syst., Aug. 2005, pp. 30873092.

[11] A. Guerra, F. Guidi, and D. Dardari, "Millimeter-wave personal radars for 3D environment mapping," in Proc. IEEE Asilomar Conf. on Signals, Systems, and Computers, Pacific Grove, USA, Nov. 2014.

[12] A. Guerra et al., "Millimeter-wave backscattering measurements with transmitarrays for personal radar applications," in Proc. IEEE Global Conf. on Commun. (GLOBECOM), LION Workshop, 2015.

[13] A. Guerra, F. Guidi, and D. Dardari, "Position and orientation error bound for wideband massive antenna arrays," in Proc. IEEE Int. Conf. on Commun. (ICC), ANLN Workshop, 2015.

[14] H. Kaouach et al., "Wideband low-loss linear and circular polarization transmit-arrays in V-band," IEEE Trans. on Antennas and Propag., vol. 59, no. 7, pp. 2513-2523, Jul. 2011.

[15] "Revision of Part 15 of the Commissions Rules Regarding Operation in the 57-64 GHz Band," Federal Communications Commission (FCC), Aug. 2013.

[16] F. Guidi et al., "Joint energy detection and massive arrays design for localization and mapping," submitted to IEEE Trans. Wireless Commun., 2016. 